\title{
Design and fabrication of Passive Fluid Driven \\ Microchannel for Fast Reaction Assays in Nano lab-on-chip Domain
}

\author{
Tijiani Adam ${ }^{1}$, U.Hashim ${ }^{1}$, Pei Ling Leow ${ }^{2}$ and S. K. Che Harun ${ }^{2}$ \\ ${ }^{1}$ Institute Nano Electronic Engineering, \\ Universiti Malaysia Perlis. (UniMAP), 01000 Kangar, Perlis Malaysia mail \\ ${ }^{2}$ Faculty of Electrical Engineering, \\ Universiti Teknologi Malaysia (UTM), 81310 Johor Bahru, Malaysia \\ tijjaniadam@yahoo.com
}

\begin{abstract}
A simple design for a microfluidic flow system for use in reacting assays with limited sample availability has been proposed, analyzed using COMSOL 3.5 Multiphysics simulation and fabricated using soft-lithography technique. The design is based on differential pressure drop flow using capillary effect concept which has facilitated a number of interesting flow phenomena in micro-domains. For an average pressure drop of about $100 / \mathrm{m}$ in the setup, flow rates of about 0.7 to $1 \mu \mathrm{l} / \mathrm{s}$ were obtained. The component consists of a microchannel, three designs were tested (50,70, 90 microns in width) to give a continuous open circuit flow. The system was designed and fabricated for continuous flow across sensing element where there is a requirement for low residence time due to fast reaction/diffusion rates.
\end{abstract}

Keywords: Microchannel, capillary, soft Lithography, Comsol Multiphysics, microfluidics

\section{Intrduction}

The integration of microfluidics with nanoelectronic sensor for the implementation of complex reaction protocols Need Capillary that has no moving parts [1], is simple and easy to implement and thus is very attractive for lowcost Microfluidic devices is very important and There is a wide spread interest in micron-scale integrated bio-molecule analysis or synthesis systems which is referred to as lab-on-a-chip $[1,2]$. A critical to this is the ability to drive a sample through the device without both moving parts and an external actuation since at the microscale level moving parts in an active mixer are very fragile. Capillarity is a force that results from the interaction of cohesion of molecules of a liquid to each other and adhesion of these molecules to the surrounding material [1-4].

\section{Methodology}

The approach presented in this paper uses a passive microfluidic capillary-driven microchannel that exploits the capillary forces to allow fluid flows: The resulting flow is driven by a combination of forces due to gravity and capillarity, the pressure and the corresponding velocity of this flow were computed using the Navier-Stokes equations for the detailed fluid motion through the device. Due to hydrostatic forces, the pressure at the inlet is given by $P=$ $\rho g \mathrm{H}$, where $\mathrm{P}$ is the pressure, $\rho$ is the density of the liquid (roughly $1000 \mathrm{~kg} / \mathrm{m} 3$ ), $g$ is the gravitational constant $(9.8 \mathrm{~m} / \mathrm{s})$, and $h$ is the height of the liquid, for water and similar substances, $\mathrm{P} \sim 10^{4} \mathrm{~h}$, with $\mathrm{P}$ in Pascal and $\mathrm{H}$ in $\mathrm{mm}$. At atmospheric pressure, $\mathrm{P} \sim 10^{5} \mathrm{~Pa}$, so $\mathrm{H}=10 \mathrm{~mm}$; If we replace the water with real sample, the effective pressure drop can be simulated since the volume of liquid that enters the channel is a negligible fraction of droplet, so that $\mathrm{H}$ is constant. In general, the pressure due to capillarity in a vertical cylindrical tube of radius $r$ can be described by (equ.1):

$P=\frac{2 \gamma(\cos \theta)}{r}$

Where is the surface tension between the liquid and air, $\theta$ is the contact angle (in radians, measured from the downward vertical between the liquid and wall, and $r$ is the radius of the inlet channel. The fluid flow tend to encounter resist due to the friction between comolecular force and adhesive force.

$\frac{\partial P}{\partial x}=\mu \frac{\partial^{2} u}{\partial y^{2}}$

Where $P$ is the applied pressure, $x$ is the dimension along the length of the channel, $\mu$ is the fluid viscosity, $u$ is the fluid particle velocity (as distinguished from the volume velocity), and $y$ is the dimension across the channel.

With no-slip boundary conditions where $\frac{\partial u}{\partial y}$ is finite so $u(0)=u(h)=0$ ), it is easy to show that $u(y)=c y(h-y)$, where $c$ is a constant. The peak velocity $u_{\max }=u(h / 2)=\operatorname{ch} 2 / 4$, so $c=$ $4 \mathrm{u}_{\max } / \mathrm{h} 2$. 


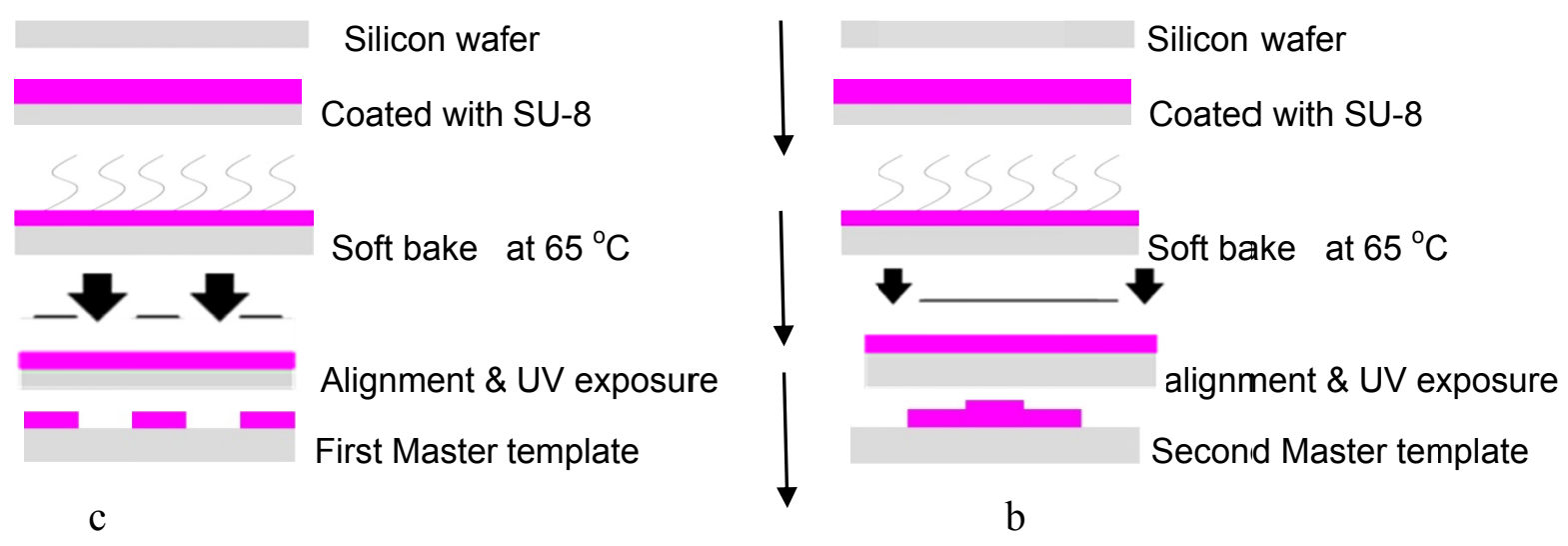

Figure2: Fabrication process flow (a) First master template (b) second Master template

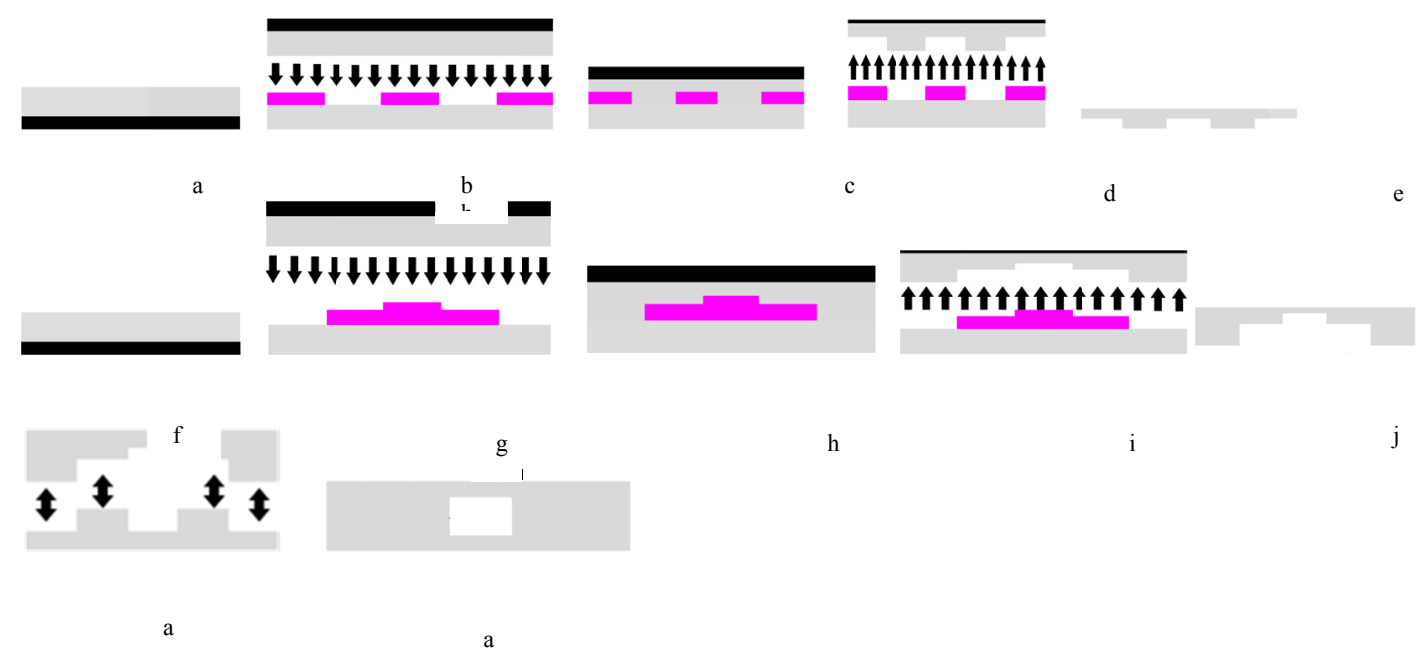

Figure3: (a) and (f) PDMS Preparation (b )and (g) PDMS was poured into master templates (c) and (g) Room temperature curing of the PDMS (d) and (i) detaches (e) and (k) fabricated devices (I) Plasma bonding (m) Bonded device[1].

\section{Results and Discussion}

A COMSOL Multiphysics software was used for Capillary flow experiments in a microchannel, the channel was formed by two parallel plates separated by distance $\mathrm{H}$. The plate separation $(H)$ is assumed to be precisely maintained throughout the length of the channel covering a range of $8 \mathrm{~mm}$ to $10 \mathrm{~mm}$ as shown in figure1c. Experiments have been conducted using Newtonian liquids (NavierStokes) for model flow. Figure 1a Shows the variation of the velocity with length of the channel, the inertia point where the first time when the fluid is injected of typical runs with unstable fluid dynamics. At the inlet, are dual forces associated in driving the fluid, a gravitation force since the inlet is vertical $(\rho \mathrm{gH})$ and the forces due capillary, in the figure $1 \mathrm{a}$, where the first point marked with pink arrow indicate the flow orientation is vertical where the velocity increases rapidly. The point shown with yellow arrow in figure1a showing distinct point where the fluid entered the channel and stabilizes for the flow, the fronts rises and stabilize and reaches the equilibrium rise height $(\mathrm{H})$ at $1.28 \mathrm{~mm} / \mathrm{s}$. The most interesting point is where a constant velocity is maintained throughout the channel length with this velocity. A sudden upshot is experience due to the fluid leaving the channel indicate by convective flux and at the $1.3 \mathrm{~mm} / \mathrm{s}$, Which is a very interesting result, to explain this, the difference in velocity between the stable state to convective influx is $0.02 \mathrm{~mm} / \mathrm{s}$ at least $4 \%$ higher. This indicates that all the fluids entered the channel leave the channel at the outlet without any disturbance hence, the channel will work perfectly without any' clog of sample. 

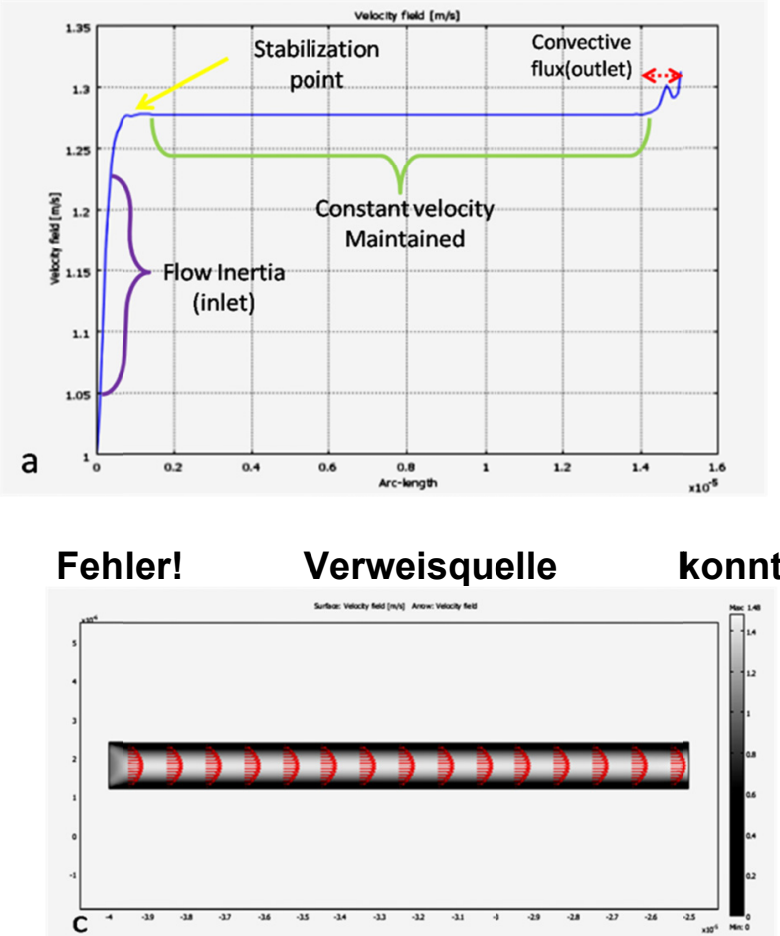

Figure1b: shows the pressure drop across the lenght of channel which fluid flow, this due to capillary forces And Capillary forces result from the interaction of liquid, gas and solid surfaces, at the interface between them. In the liquid phase, molecules are held together by cohesive forces. In the bulk of the liquid, the cohesive forces between one molecule and the surrounding molecules are balanced. However, for the same molecule at the edge of the liquid, the cohesive forces with other liquid molecules are larger than the interaction with air molecules, As a result, the liquid molecules at the interface are pulled together towards the

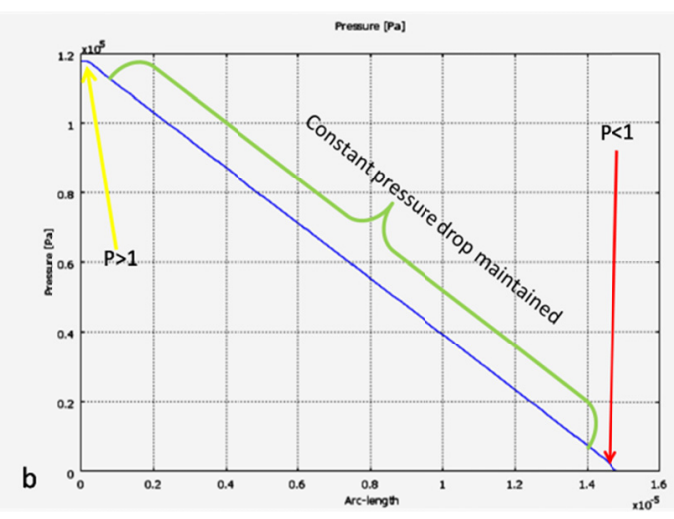

nicht gefunden werden. liquid, here in this experiments, and the effect of the pressure could be explained in three major points within the microchannel. At the inlet where the pressure must be greater than every point before subsequent point ahead for the fluid to start flow and in this case is greater than unity but here, the value is chosen for the purpose of explanation since the pressure used here is atmospheric pressure and this will save the purpose. The yellow arrow indicates the point at which the pressure the fluid started flowing 1.1x10E5 $(\mathrm{Pa})$ and from there the pressure gradually drops to zero with constant value as the fluid flows.
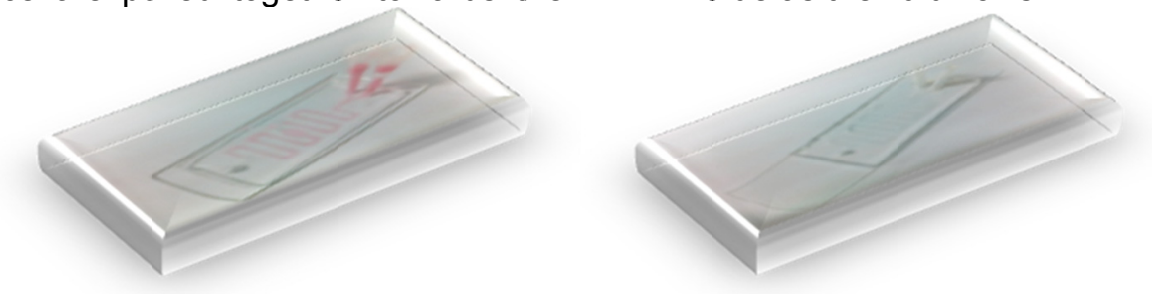

Figure2: shows the fabricated microchannels
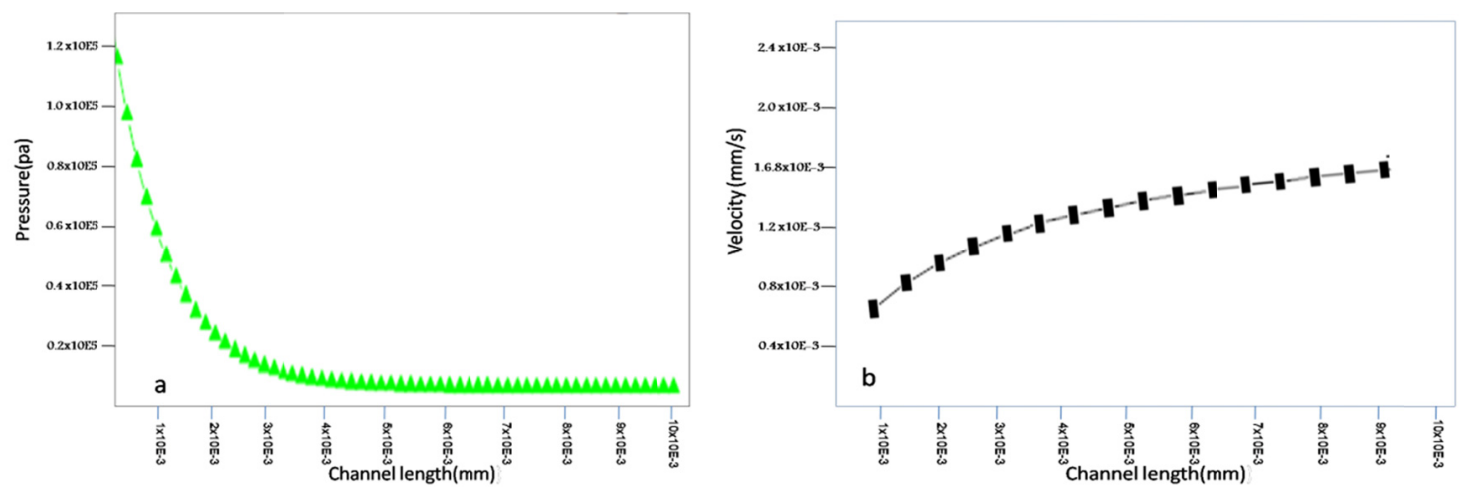

Figure3: Shows (a) the drop in pressure as the fluid flow through the channel (b) the velocity trend of the fluid as the fluid flows. 
Figure3 shows the experimental result obtained during testing on the fabricated micro channel, Its can be seen that from figure 1 band $3 a$, the graph differ a bit, the graph for the figure $1 b$ drop linearly but the graph for the figure $3 a$ not a linear one but still suggest that the pressure is dropping and likewise, the velocity curve shown little disperaty, this can be seen in figure $1 a$ and figure $3 b$, this might be due some error in the measurement instrument or human error.

\section{Conclusion}

Capillary effect for driving a fluid within microchannel have be reported. Both the numerical analysis and the experimental analysis were performed, each provided an inside to the flow dynamics of sample in a microchannel due, when $70 \mu \mathrm{m}$ channel was design and fabricated, a uniform pressure drops and constant velocity were observed, using this phenomena both approaches provided similar results with this demonstrate the potential of using this phenomena to employ for passive fluid control within microchannel which a fluid could flow passively without any external force.

\section{Acknowledgment}

The authors wish to thank Universiti Malaysia Perlis (UniMAP and Ministry of Higher Education Malaysia for giving FRGS grant to conduct this research in the Micro \& Nano Fabrication Lab. Appreciation also goes to all the team members in the Institute of Nanoelectronic Engineering especially the Nano structure Lab On chip Research Group.

\section{Reference}

[1] Tijjani Adam, U.Hashim, Pei Ling Leow , Pei Song Chee and K. L. Foo "Fabrication of PDMS multi-layer microstructure: The electroosmosis mechanism in fluidics for life sciences "Enabling Science and Nanotechnology (Escinano), 2012 International Conference, 5-7 Jan. 2012, pp 1 - 410.1109/ESciNano. 2012.6149698.

[2] Kuo-Kang Liu ,Ren-Guei Wu,Yun-Ju Chuang, Hwa Seng Khoo, Shih-Hao Huang and Fan-Gang Tseng (2010)“ Microfluidic Systems for Biosensing" Sensors 2010, 10, 6623-6661; doi:10.3390/s100706623

[3] Park, S.Y.; Kalim, S.; Callahan, C.; Teitell, M.A.; Chiou, E.P.Y. A light-induced dielectrophoretic droplet manipulation platform. Lab Chip. 2009, 9, 3228-3235.
[4] Sato, K.; Mawatari, K.; Kitamori, T. Microchip-based cell analysis and clinical diagnosis system. Lab Chip. 2008, 8, 19921998. 